\title{
Repercusiones biopsicosociales en los padres que tienen un hijo con hemofilia
}

\author{
Biopsychosocial repercussions in parents who have a child with hemophilia
}

\author{
Maricela Osorio G. ${ }^{1}$ \\ Universidad Nacional Autónoma de México, Facultad de Estudios Superiores Iztacala.
}

RECIBIDO 01/09/2016, AcEPTADO 28/10/2016

\begin{abstract}
RESUMEN
Recibir el diagnóstico de un padecimiento crónico en familia trae consigo cambios y repercusiones drásticas. La hemofilia es una enfermedad hemorrágica crónica hereditaria que necesita de cuidados específicos. El objetivo de este trabajo fue analizar y describir el impacto familiar multidimensional del diagnóstico de hemofilia en pacientes pediátricos en un grupo de progenitores. Participaron 20 padres (10 varones y 10 mujeres), respondiendo el instrumento PedsQL ${ }^{\mathrm{TM}}$ 2.0, Módulo de Impacto familiar desarrollado por Varni et al. (2004). Las puntuaciones generales bajas indican una afectación biopsicosocial de los padres; el área más afectada es la de preocupación y las principales repercusiones físicas son insomnio, dolores de cabeza y de estómago. Es necesario evidenciar estos problemas y diseñar programas de atención integral para padres.
\end{abstract}

Palabras clave: Repercusiones biopsicosociales; familia; hemofilia; enfermedades crónicas.

\begin{abstract}
Receiving a diagnosis of a chronic condition in family, brings changes and drastic repercussions. Hemophilia is an hereditary chronic hemorrhagic disease that needs specific care The objective of this study was to analyze and describe the multidimensional family impact of the hemophilia diagnosis in pediatric patients in a group of progenitors. Attended by 20 parents (10 males and 10 females) answering the PedsQL ${ }^{\mathrm{TM}} 2.0$ instrument. The Family Impact Module developed by Varni et al., (2004). The low overall scores indicate a biopsychosocial affectation of the parents; the most affected area is the concern and the main physical repercussions are : insomnia, headaches and stomach ache. It is necessary to highlight these problems and design integral care programs for parents.
\end{abstract}

Keywords: Biopsychosocial repercussions, family, hemophilia, chronic diseases.

1 Profesora e investigadora de la Carrera de Psicología UNAM. Facultad de Estudios Profesionales Iztacala. Doctorado en Ciencias Psicológicas y Pedagógicas, Área: Psicología de la Salud y Prevención del Riesgo Individual y Social por la Universidad de los Estudios de Nápoles Federico Il. Participa en la línea de investigación: Enfermedades crónicas. Pertenece al Sistema Nacional de Investigadores (SNI). E-mail: mosguz@gmail.com 


\section{INTRODUCCIÓN}

Los logros espectaculares en las ciencias de la salud han conseguido grandes avances en la detección, tratamiento y control de las enfermedades. Lo anterior ha permitido un incremento visible en la expectativa de vida de pacientes con enfermedades crónicas no transmisibles (Osorio, Marín, Bazán \& Ruíz, 2013).

Estas enfermedades constituyen un problema de salud en las sociedades contemporáneas, y el aumento sostenido de la incidencia y prevalencia de las mismas a nivel mundial, desde el pasado siglo, las ha convertido en un verdadero reto económico, político, social y personal (Ledón, 2011; Osorio \& Graña 2016).

Según Roca y Pérez (2010), las enfermedades crónicas rebasan los marcos institucionales sanitarios y abarcan la vida cotidiana de la persona, así como sus redes sociales. Ledón (2011) sostiene que este tipo de padecimiento, una vez instaurado, puede producir cambios psíquicos y corporales intensos y modificar áreas de vida significativas, lo cual demanda de las ciencias de la salud un enfoque holístico al problema.

Así, cuando una familia recibe la noticia de que uno de sus miembros tiene alguna enfermedad crónica, se producen cambios estructurales, procesuales y emocionales en la misma (Grau y Fernández, 2010). Estas reacciones dependen de numerosos factores como experiencia en situaciones de crisis y problemas médicos, estatus socio-económico, nivel de conocimientos, calidad de los servicios sanitarios y educativos y sistemas de apoyo. La familia es entonces uno de los espacios de vida más profundamente impactados.

Cuando un hijo en edad pediátrica es afectado con un diagnóstico de este tipo, la familia es la primera red de apoyo social y la que ejerce una función protectora (Ledón, 2011; Reyes, Garrido, Torres, Ortega, 2010). La enfermedad del niño es siempre un problema familiar y su calidad de vida está íntimamente relacionada con el apoyo que recibe.

Los padres responderán no solo en función de la enfermedad y del tratamiento del niño, sino también según sus propios sentimientos y problemas personales, lo que significa que tanto las reacciones del niño enfermo como la de sus padres son interdependientes (Grau y Fernández, 2010).

Los padres tienen que reestructurar todos los aspectos de la vida familiar y la mayoría de las veces lo hacen en función de la enfermedad del hijo, olvidándose en muchas ocasiones de otros aspectos importantes de su vida, ya que la amenaza que representa el padecimiento absorbe todo su tiempo, atención y energía. Algunos autores han descrito que la enfermedad crónica produce cambios en la mayoría de las rutinas familiares, por ejemplo, González, Steinglass y Reiss (1987) mencionan que se produce una acomodación a las necesidades de la enfermedad, y las rutinas familiares se reestructuran (por ejemplo, uno de los padres deja de trabajar para hacerse cargo del cuidado del niño), añaden que las decisiones relacionadas con el ciclo vital familiar y con el desarrollo individual de los miembros de la familia quedan postergadas y que suele aparecer un desequilibrio en la distribución de 
recursos, además de que la identidad familiar se distorsiona, ya que la enfermedad es su principal organizador. Para Reyes et al. (2010), existe una redistribución de los gastos familiares, porque se incrementa la compra de medicamentos, mencionan también que hay modificaciones en los horarios de comidas, las salidas o los lugares frecuentados, y cambios en la convivencia familiar. Por su parte, Grau y Fernández (2010) mencionan que se producen cambios en la asignación de papeles, la división del trabajo y las actividades recreativas o celebraciones festivas, los planes y prioridades de las familias y el modo en que se expresan las emociones.

A nivel personal, las enfermedades crónicas tienen repercusiones en prácticamente todos los aspectos biosicosociales del individuo con trascendencia también sobre la pareja (Torres y Gutiérrez, 2010).

Esto es especialmente cierto en las parejas en las que hay algún miembro diagnosticado con hemofilia, debido a que padecer una enfermedad crónica como esta requiere cuidados, tratamientos, información, capacitación y apoyos especiales (Osorio, Luque, Bazán y Gaitán, 2012).

La hemofilia es una enfermedad crónica hereditaria de carácter recesivo, no contagiosa y ligada al sexo, que se caracteriza por la insuficiencia de uno o más factores necesarios para la coagulación sanguínea (factor VIII, para la hemofilia A o factor IX, para la hemofilia B). Se clasifica en grave, moderada o leve, en función del nivel de deficiencia del factor de coagulación y se estima que a nivel mundial afecta a 1 de cada 5000 varones nacidos vivos (Pruthi, 2005). Su evolución está caracterizada por las complicaciones ocasionadas por hemorragias recurrentes que originan discapacidad física y que en muchas ocasiones ponen en riesgo la vida de los pacientes (Osorio, et al., 2013). Los sangrados más frecuentes son internos y pueden no ser visibles, afectando principalmente articulaciones como las rodillas, los tobillos y los codos. Estos sangrados provocan dolor muy intenso e inflamación y cuando no se tratan oportuna y adecuadamente provocan discapacidades severas, afectando de por vida al paciente. Además, hay sangrados que ponen en riesgo su vida, como los del sistema nervioso central (SNC).

La enfermedad, sin un tratamiento adecuado, provoca limitación en las actividades; continuas e inesperadas hospitalizaciones o visitas ambulatorias frecuentes, también requiere de cuidados específicos diarios, lo que conlleva a que no siempre las personas que la padecen puedan participar en actividades cotidianas de compañeros o coetáneos ocasionando, según autores como Jones (2004); Sarmiento, Carruyo, Carrizo, Vizcaíno, Arteaga y Vizcaíno (2006); y Carruyo, Vizcaíno, y Carrizo, (2004), dificultad en su crecimiento y desarrollo integral. Debido a que la hemofilia no afecta únicamente al paciente sino a su entorno más cercano, a toda la familia y especialmente los padres, estos se ven afectados por lo anteriormente descrito.

En México, la Federación de Hemofilia de la República Mexicana A.C. (Reporte sobre hemofilia en México, 2016) informa que existen 5221 pacientes detectados, de los cuales hasta el momento no se tiene un informe detallado de su estado integral de salud ni de sus niveles de bienestar. 
La calidad de vida (CV) es entendida como un componente central del bienestar humano, relacionado con aspectos de su funcionamiento como la salud y el desarrollo de habilidades de interacción interpersonales o sociales en la familia, en la escuela, en el trabajo o en la comunidad. Recientemente, se ha introducido el concepto Calidad de Vida Relacionado con la Salud (CVRS), el cual llegó al campo sanitario con la mirada de que el bienestar de los pacientes es un punto importante de ser considerado tanto en su tratamiento como en todo el proceso de salud-enfermedad. La CV se diferencia de la CVRS en que este último término es utilizado en el área sanitaria y evalúa la calidad de los cambios resultado de las intervenciones del equipo de salud, mide la experiencia que el paciente tiene de su enfermedad, su experiencia respecto a los cuidados médicos y determina el impacto de la enfermedad en su vida diaria, es decir, es una medición de salud desde la perspectiva de los pacientes (Haas, 1999; Schwartzmann, 2003).

De esta manera, y partiendo del hecho de que la perspectiva del paciente y su familia es fundamental para tener un conocimiento objetivo de la afectación del padecimiento, se han desarrollado instrumentos que evalúan la CVRS y sus repercusiones en el ámbito familiar, tal es el caso del PedsQL ${ }^{\text {TM }}$ 2.0, Módulo de Impacto familiar desarrollado por Varni Sherman, Burwinkle, Dickinson, Dixon (2004) instrumento utilizado en este estudio, con autorización del autor.

En el presente trabajo, se analizaron las repercusiones biopsicosociales en los progenitores de pacientes pediátricos con hemofilia, analizando ocho áreas importantes en la medición del impacto familiar. Se seleccionaron 20 progenitores de pacientes con niños pequeños (de 2 a 5 años de edad), los cuales según la literatura son aquellos con mayores problemas y repercusiones en su funcionamiento familiar y de pareja.

Debido a lo anterior, se decidió hacer una evaluación de los efectos del padecimiento en este grupo de progenitores, por lo que el objetivo del presente trabajo fue analizar y describir el impacto familiar multidimensional del diagnóstico de hemofilia en pacientes pediátricos en un grupo de progenitores.

\section{MÉTODO}

\section{Participantes}

Participaron 20 progenitores de niños diagnosticados con hemofilia, 10 varones y 10 mujeres, cuyas edades iban de 22 a 38 años $(X=29.25$; D.T= 5.11; rango= 16). Respecto a su escolaridad, el 15\% tenía estudios de primaria, el $40 \%$ de secundaria, otro $40 \%$ preparatoria o alguna carrera técnica y el 5\% tenía estudios universitarios.

\section{Instrumento}

Se utilizó el PedsQL ${ }^{\text {TM }}$ 2.0, Módulo de Impacto familiar desarrollado por Varni et al., (2004). El instrumento fue diseñado a base de un modelo multidimensional y 
modular que incluye las dimensiones sugeridas por la Organización Mundial de la Salud para la Evaluación de la Calidad de Vida (WHOQoL,1995).

El PedsQL ${ }^{\mathrm{TM}}$ Módulo de Impacto Familiar está constituido por 36 ítems divididos en 6 escalas que miden el auto-reporte de la funcionalidad de los padres: 1) Funcionamiento Físico Salud (6 ítems); 2) Funcionamiento Emocional (5 ítems); 3) Funcionamiento Social (4 ítems); 4) Funcionamiento Cognitivo (5 ítems); 5) Comunicación (3 ítems); 6) Preocupaciones (5 ítems); y 2 escalas que miden la funcionalidad familiar reportada por los progenitores; 7) Actividades Diarias (3 ítems); y 8) Relaciones familiares (5 ítems). El instrumento presenta 5 posibilidades de respuesta (de $0=$ nunca es un problema a $4=$ siempre es un problema). Los ítems se califican de forma inversa y se transforman linealmente en una escala de 0 a $100(0=100,1=75,2=50,3=25,4=0)$, de modo que una mayor puntuación indica un mejor funcionamiento (Fairclough, 2002). El instrumento tiene una confiabilidad de 0.90 .

Existen tres medidas que se pueden obtener del instrumento: A) El Puntaje total del Impacto, el cual se obtiene sumando los 36 ítems del test; B) El Puntaje del Impacto en la Calidad de Vida relacionado con la salud (HRQOL), el cual incluye los 20 ítems de las primeras 4 escalas y C) El Puntaje en el área de repercusiones familiares, en el cual se suman los 8 ítems de las dos últimas escalas (Varni Sherman, Burwinkle, Dickinson, Dixon, 2004).

Los instrumentos fueron aplicados en el auditorio de un hospital público y en la Casa de la Hemofilia, ambos ubicados en la Ciudad de México. Todos los progenitores firmaron el consentimiento informado. El proyecto general del cual se desprende esta investigación fue revisado y aprobado por el Comité de Bioética de la Carrera de Psicología de la Facultad de Estudios Superiores Iztacala.

\section{Procedimiento}

Una vez seleccionada la muestra, se pidió que leyeran y firmaran el consentimiento informado; posteriormente, se les aplicó el cuestionario de manera individual. Se diseñó la base de datos en el programa SPSS 20 y se procedió al análisis.

\section{Análisis de datos}

Se realizó un análisis de tipo descriptivo, que diera cuenta puntualmente de las características de la muestra. Se calcularon los totales por escala y se obtuvieron los índices de correlación ( $\mathrm{r}$ de Pearson) entre los totales de cada una de ellas y las variables demográficas. 


\section{RESULTADOS}

\section{Características demográficas de la muestra}

Participaron 20 progenitores de niños diagnosticados con hemofilia, (10 varones y 10 mujeres). La mayoría de ellos llevaba poco tiempo de haberse incorporado a la Federación de Hemofilia y había asistido 1 o 2 veces a las reuniones de dicha Asociación.

La edad promedio de sus hijos fue de 3.7 años (D.T.=1.1); el tipo de hemofilia que padecían los niños era el $90 \%$ hemofilia A y el restante $10 \%$ hemofilia $\mathrm{B}$. Respecto del grado clínico, el 25\% era leve, el 10\% moderado y el 65\% severo. El 90\% de ellos eran hijos únicos.

El $100 \%$ de los varones declararon tener una actividad laboral remunerada y solo el 20\% de las mujeres manifestaron trabajar fuera de casa. 30\% de los participantes manifestó estar separada en ese momento de su pareja debido a problemas conyugales.

\section{Impacto familiar de la hemofilia}

De la aplicación del PedsQL ${ }^{\text {TM }}$ Módulo de Impacto Familiar, se desprende que los niveles de calidad de vida generales de las familias participantes en el presente estudio son bajos, lo que implica poca funcionalidad de los padres en las diferentes áreas. Como se puede observar en la tabla 1, el puntaje total es bastante bajo $(55 / 100)$, lo que indica que los padres no han tenido una buena adaptación y que hay repercusiones fácilmente identificables. Aunque el Índice general de impacto en la calidad de vida relacionada con la salud de los padres es mayor que el puntaje anterior (60.5/100), este también denota una afectación a nivel biopsicosocial de los padres como protagonistas del proceso mórbido por el cual cursan sus hijos. Una de las áreas más afectadas, es la familiar con la media más baja (53.3/100), dato que revela problemas en el desempeño de las actividades diarias y en las relaciones de los miembros de este grupo primario.

Analizando más detalladamente la tabla 1, se puede observar que la sub-escala con el puntaje más bajo es la de preocupación, la cual hace referencia en términos generales al desasosiego que generan los efectos secundarios del tratamiento y su medicación (ver tabla 1).

La otra área con bajos puntajes tiene que ver con la salud física de los progenitores, los cuales, al enfrentarse con un padecimiento como la hemofilia, sufren repercusiones en su propia salud, como insomnio, dolores de cabeza o de estómago, etc. 
Tabla 1.Puntajes en cada una de las escalas del PedsQL ${ }^{\mathrm{TM}}$ Módulo de Impacto Familiar

\begin{tabular}{lcccc}
\hline \multicolumn{1}{c}{ Sub-escalas } & \# ítems & $\mathrm{N}$ & Media & D.T \\
\hline Puntaje total del impacto & 36 & 20 & 55.1 & 8.8 \\
Puntaje calidad de vida relacionada con la salud & 20 & 20 & 60.5 & 7.5 \\
& & & & \\
Salud y actividades físicas & 6 & 20 & 51.7 & 14.8 \\
Estado emocional & 5 & 20 & 61 & 12.2 \\
Actividades sociales & 4 & 20 & 59.1 & 16.5 \\
Pensamiento y concentración & 5 & 20 & 71.5 & 17 \\
Comunicación & 3 & 20 & 50 & 20.6 \\
Preocupación & 5 & 20 & 39.5 & 15.3 \\
& & & & \\
Puntaje familiar & 8 & 20 & 53.3 & 16.3 \\
Actividades diarias & 3 & 20 & 48.3 & 27.4 \\
Relaciones familiares & 5 & 20 & 56.3 & 22.5 \\
\hline
\end{tabular}

Como se puede observar en la tabla 2, existen diferencias (aunque estas no son estadísticamente significativas) en las medias de las puntuaciones que presentan padres y madres, siendo los padres los que tienen índices más altos, lo que implica que son los varones quienes están aparentemente mejor adaptados al padecimiento de su hijo.

Tabla 2. Medias obtenidas por padres y madres en los puntajes de las 3 escalas generales del

$$
\text { PedsQL }{ }^{\mathrm{TM}}
$$

\begin{tabular}{llllc}
\hline \multicolumn{1}{c}{ Puntuación } & Género & $\mathrm{N}$ & Media & $\mathrm{DT}$ \\
\hline \multirow{2}{*}{ Total impacto } & Masculino & 10 & 55.7 & 9.6 \\
& Femenino & 10 & 54.4 & 8.3 \\
\hline \multirow{2}{*}{ Total CVRS padres } & Masculino & 10 & 61.3 & 8.8 \\
& Femenino & 10 & 59.5 & 6 \\
\hline \multirow{2}{*}{ Total familia } & Masculino & 10 & 53.4 & 15.8 \\
& Femenino & 10 & 53.1 & 17.6 \\
\hline
\end{tabular}

Del análisis estadístico aplicado para ver si existían diferencias significativas por género en las diversas áreas del instrumento, se obtuvo que existen solamente en dos de ellas: en la escala de salud $(\mathrm{t}=4.4 ; \mathrm{gl}=18 ; \mathrm{p}<0.00)$ y en la escala de actividades diarias $(\mathrm{t}=2.24 ; \mathrm{gl}=18 ; \mathrm{p}<0.03)$. En ambos casos son las madres quienes obtuvieron las medias más bajas, es decir, aquellas con mayores repercusiones. 


\section{Correlaciones entre variables}

Respecto de la asociación entre las variables demográficas y las escalas, se encontró que existe una correlación fuerte significativa ente el género femenino y las repercusiones en la salud $(\mathrm{r}=0.72 ; \mathrm{p}<0.00)$; y una correlación moderada entre el ser mujer y la dificultad en llevar a cabo las actividades diarias $(r=0.47 ; \mathrm{p}<0.05)$; lo anterior significa que son las mujeres aquellas que manifiestan tener mayores efectos en su salud física y las que reportan un mayor esfuerzo en llevar a cabo las tareas diarias de la casa.

Se encontraron, también, correlaciones moderadas significativas entre las escalas salud y comunicación, con la edad del padre $(r=0.47 ; \mathrm{p}<0.05$ y $\mathrm{r}=0.48 ; \mathrm{p}<0.05$, respectivamente). Índices que denotan que a mayor edad de los padres hay más afectación en la salud de los progenitores y también que a mayor edad de los padres habrá mejor comunicación entre la pareja.

Una última asociación entre las variables fue la encontrada entre la escala de preocupación y la edad del niño. Dicha correlación fue moderada significativa y negativa $(r=-0.49 ; p<0.05)$, lo cual indica que habrá mayores índices de preocupación cuando el paciente pediátrico tenga menor edad.

\section{Análisis descriptivo de los datos}

A partir de los datos obtenidos en cada una de las escalas del instrumento aplicado, se puede deducir que una de las repercusiones principales en la familia de un paciente pediátrico con hemofilia es la preocupación que manifiestan ambos padres respecto del futuro del hijo; los participantes también declararon que les preocupa si los tratamientos médicos del hijo están funcionando y, a su vez, les inquietan los efectos secundarios del tratamiento y la medicación.

En el área de actividades diarias, las mujeres manifestaron sentirse demasiado cansadas para terminar las tareas de la casa, así como la convicción de que las actividades familiares llevan más tiempo y esfuerzo debido a la enfermedad de su hijo. En la escala de comunicación, los progenitores expresaron que les cuesta hablar de la salud de su hijo con los demás y que sienten que los otros no entienden su situación familiar.

Respecto de la escala de Salud, reportaron sentirse cansados durante el día, tener dolores de cabeza con frecuencia y sentirse físicamente débiles. Es necesario hacer mención que son las mujeres quienes obtuvieron porcentajes más altos en la sintomatología reportada.

Otra de las áreas afectadas son las relaciones familiares, en donde los participantes manifestaron dificultades en tomar decisiones en conjunto como familia, una ausencia de comunicación entre sus miembros y la dificultad en resolver problemas familiares juntos.

Las actividades sociales también se ven afectadas ya que los progenitores reportaron sentirse aislados de los demás, no tener suficiente energía para las actividades sociales y tener problemas en conseguir apoyo de los demás. 
Finalmente, respecto a su estado emocional manifestaron sentirse enojados, tristes, ansiosos, frustrados y desamparados.

\section{DISCUSIÓN Y CONCLUSIONES}

El diagnóstico de una enfermedad crónica en pacientes pediátricos repercute indudablemente no solo en la rutina diaria de la familia, sino directamente en la calidad de vida de los progenitores del paciente. Como se muestra en la presente investigación, existen repercusiones a nivel biopsicosocial, las cuales deben ser reconocidas y mostradas para proponer soluciones.

Más allá de los cambios y modificaciones en los roles de la familia de un enfermo crónico, estudios como el presente evidencian las repercusiones directas que hay en la salud física, el estado emocional, el área social, de comunicación y familiar de los progenitores.

Estos efectos negativos son factores que influyen, a su vez, en la convivencia con la pareja, ya que un porcentaje considerable de las personas que participaron manifestó que su relación estaba sumamente afectada por el exceso de cansancio, también exteriorizaron molestias físicas y psicológicas por la falta de apoyo en las actividades cotidianas del hogar, así como sentirse con poca energía para frecuentar junto con la pareja actividades sociales.

Como se mencionó en la parte introductoria del presente trabajo, muchas de las reacciones de los padres dependen de diferentes factores (Grau y Fernández, 2010). En el presente estudio, características como la edad del niño, la reciente incorporación a las actividades de la Federación -lo que implica poca interacción con familias y pacientes con la enfermedad, así como poca información sobre el padecimiento- los altos niveles de preocupación y los problemas de separación con sus respectivas parejas influyeron en que haya un alto impacto familiar de la enfermedad. Es evidente entonces que, como lo mencionan algunos autores, la identidad de estas familias se distorsiona, ya que la enfermedad se vuelve su principal organizador (González, Steinglass y Reiss, 1987).

Respecto de las correlaciones encontradas, es importante mencionar el hecho de que, en esta muestra, la distribución de las actividades está determinada por roles estereotipados de género, ya que las mujeres resultaron asociadas significativamente con el área de actividades en el hogar, escala en la cual los varones no reportan problema alguno. Es también interesante resaltar que, según los resultados, son las madres las que tienen niveles más altos de afectación en su salud física.

Aunque los resultados del presente estudio se consideran relevantes, se piensa que se debe ampliar la muestra, así como aplicar instrumentos que indaguen otros aspectos individuales y grupales de este tipo de familias, para comparar variables diferentes.

A partir de lo aquí expuesto se propondrán diversas estrategias de intervención, como un taller donde se aprendan diferentes técnicas de relajación para el manejo 
de la ansiedad y la preocupación que genera el tener a cargo un paciente con hemofilia, un programa de entrenamiento en habilidades sociales, para, entre otras cosas, mejorar la comunicación de los miembros de la familia y personas externas a ella. Además, es necesario difundir y entender que la hemofilia no solo afecta a la persona que la padece, sino a todos los miembros de la familia, por lo cual la atención de los profesionistas de la salud se debe ampliar a ellos. Como recomendación, es necesario animar a los padres a que participen regularmente en grupos de apoyo u organizaciones de hemofilia, donde puedan hablar sobre dudas y temores con otros padres cuyos hijos estén afectados por el mismo padecimiento, lo cual seguramente hará que su experiencia con la enfermedad se enriquezca y que sus niveles de preocupación disminuyan.

\section{REFERENCIAS BIBLIOGRÁFICAS}

Carruyo, V.C, Vizcaino G., y Carrizo, E. (2004). Actitud de los individuos adultos con hemofilia hacia su enfermedad. Invest. Clín; 45:257-267. Recuperado el 18/01/2016 Dirección URL: http://www.scielo.org.ve/scielo. php?script=sci_arttext\&pid=S0535-51332004000300006

Fairclough, D. L. (2002). Design and Analysis of Quality of Life Studies in Clinical Trials: Interdisciplinary Statistics. New York: Chapman \& Hall/CRC.

González, S., Steinglass, P., y Reiss, D. (1987). Intervenciones centradas en familias con miembros que tienen dolencias crónicas. USA: George Washington University.

Grau, C. y Fernández H. M. (2010). Familia y enfermedad crónica pediátrica An. Sist. Sanit. Navar. 2010; 33 (2): 203-212. Recuperado el 18/01/2015 Dirección URL: http://scielo.isciii.es/scielo.php?pid=S1137$66272010000300008 \&$ script $=$ sci_arttext\&tlng $=$ pt

Haas, B. (1999). Clarification and integration of similar Quality of life concepts. Journal of Nursing Scholarships. 31: 215-20. Recuperado el 18/01/2016 Dirección URL: http://www.ncbi.nlm.nih.gov/pubmed/10528449

Jones, P. (2004). Living with Heamophilia. USA: Oxford University Press.

Ledón, Ll. L. (2011). Enfermedades crónicas y vida cotidiana. Revista Cubana de Salud Pública; 37(4):488-499. Recuperado el 18/01/2016 Dirección URL: http://www.redalyc.org/articulo.oa?id=21421364013

Osorio, G.M. Marín, P.T. Bazán, G. y Ruiz, N. (2013). Calidad de vida de pacientes pediátricos con hemofilia. Consulta externa de un hospital público de la Ciudad de México. Revista de Psicología GEPU 4(1) 13-26. Recuperado 
el 18/01/2016 Dirección URL: http://revistadepsicologiagepu.es.t1/Calidadde-Vida-de-Pacientes-Pedi\%E1 tricos-con-Hemofilia-.--Consulta-Externade-un-Hospital-P\%FAblico-de-la-Ciudad-de-M-e2-xico.htm

Osorio, G.M., Luque, M., Bazán, G. y Gaitán C. (2012). Propiedades psicométricas del cuestionario calidad de vida en pacientes pediátricos con hemofilia México (QoLHMEX). Revista Electrónica de Psicología Iztacala; 15 (3), 1112-11128. Recuperado el 18/01/2016. Dirección URL: http://www. revistas.unam.mx/index.php/repi/article/view/33647

Pruthi, R. K. (2005). Hemophilia: a practical approach to genetic testing. Mayo Clin Proc; 80(11):1485-99.

Reyes L., A.G., Garrido G., A., Torres, V., L.E. y Ortega, S. P. (2010). Cambios en la cotidianidad familiar por enfermedades crónicas. Psicología y Salud, 20 (1): 111-117. Recuperado el 18/01/2016 Dirección URL: www.uv.mx/ psicysalud/psicysalud-20-1/20-1/adriana-guadalupe-reyes-luna.pdf

Roca, M. y Pérez, M. (2000). Apoyo Social. Su significación para la Salud Humana. La Habana, Cuba: Editorial Félix Varela

Sarmiento, S. Carruyo, C. Carrizo, E. Arteaga, M. V. y Vizcaíno, G. (2006). Funcionamiento social en niños hemofílicos. Análisis de encuesta para determinar factores psicopatológicos de riesgo. Rev Méd Chile, 134:5359. Recuperado el 18/01/2016 Dirección URL: http://www.scielo.cl/scielo. php?script=sci_arttext\&pid=S0034-98872006000100007

Schwartzmann, L. (2003). Calidad de vida relacionada con la salud: Aspectos conceptuales. Ciencia y Enfermería; 9: 9-21. Recuperado el 18/01/2016 Dirección URL: http://www.scielo.cl/scielo.php?script=sci_arttext\&pid $=$ S0717-95532003000200002

Torres, R. B. y Gutiérrez, G. C. (2010). Enfermedad crónica, calidad de vida y sexualidad. Investigaciones Medicoquirúrgicas. 2 (2): 23-29. Recuperado el 18/01/2016 Dirección URL: http://www.revcimeq.sld.cu/index.php/imq/ article/view/49

Varni, W. J., Sherman, S. A., Burwinkle, T. M., Dickinson, P.E. y Dixon, P. (2004). The PedsQL ${ }^{\mathrm{TM}}$ Family Impact Module: Preliminary reliability and validity. Health and Quality of Life Outcomes, 2:55. Doi:10.1186/1477-7525-2-55

WHOQOL Group (1995). World Health Organization Quality of Life Assessment: position paper from the World Health Organization. Soc Sci Med 41: 1403. Recuperado el 18/01/2016 Dirección URL: http://www.ncbi.nlm.nih.gov/ pubmed/ 8560308 